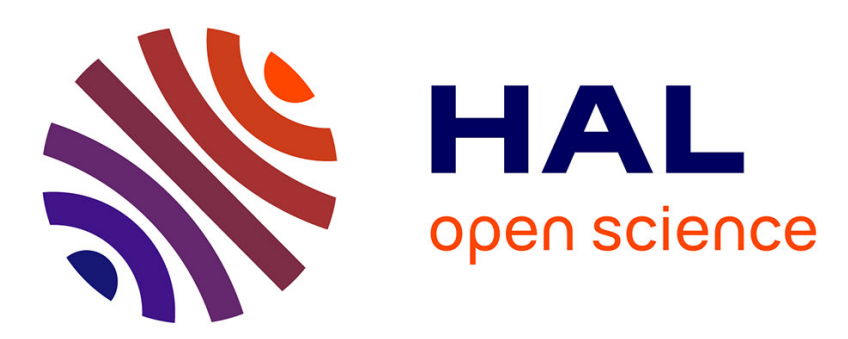

\title{
A Collaborative Methodology for Tacit Knowledge Management: Application to Scientific Research
}

\author{
Emna Mezghani, Ernesto Expósito, Khalil Drira
}

\section{To cite this version:}

Emna Mezghani, Ernesto Expósito, Khalil Drira. A Collaborative Methodology for Tacit Knowledge Management: Application to Scientific Research. Future Generation Computer Systems, 2016, 54, pp. 450-455. 10.1016/j.future.2015.05.007 . hal-01149983

\section{HAL Id: hal-01149983 https://hal.science/hal-01149983}

Submitted on 8 May 2015

HAL is a multi-disciplinary open access archive for the deposit and dissemination of scientific research documents, whether they are published or not. The documents may come from teaching and research institutions in France or abroad, or from public or private research centers.
L'archive ouverte pluridisciplinaire HAL, est destinée au dépôt et à la diffusion de documents scientifiques de niveau recherche, publiés ou non, émanant des établissements d'enseignement et de recherche français ou étrangers, des laboratoires publics ou privés. 


\title{
A Collaborative Methodology for Tacit Knowledge Management: Application to Scientific Research
}

\author{
Emna MEZGHANI $^{\mathrm{a}, \mathrm{b}, \mathrm{c}, \mathrm{d}, 1}$, Ernesto EXPOSITO ${ }^{\mathrm{a}, \mathrm{c}}$, Khalil DRIRA $^{\mathrm{a}, \mathrm{b}}$ \\ ${ }^{\text {a }}$ CNRS, LAAS, 7 avenue du colonel Roche, F-31400 Toulouse, France \\ ${ }^{\mathrm{b}}$ Univ de Toulouse, LAAS, F-31400 Toulouse, France \\ ${ }^{\mathrm{c}}$ Univ de Toulouse, LAAS, INSA, F-31400 Toulouse, France \\ ${ }^{\mathrm{d}}$ Luxembourg Institute of Science and Technology, 5, Avenue des Hauts-Fourneaux, L-4362, \\ Esch/Alzette, Luxembourg \\ \{emna.mezghani, ernesto.exposito, khalil.drira\}@laas.fr
}

\begin{abstract}
Tacit knowledge, which refers to the know-how, is critical to understand and reuse since it is located in the human heads. It represents the foremost element for human and team evaluation. Seeking for tacit knowledge is achieved only by communicating with the concerned persons, which makes losing it axiomatic if people leave their work without documenting their known-how. Thus, providing a collaborative environment based on a common conceptualization of the domain to formalize the experts' knowledge and to share their outcomes is required. However, some barriers pertaining to cultural and social factors such as personality traits impede capturing the conceptual model. To cope with these issues, we have proposed a generic two-step methodology that copes with human barriers when capturing the domain experts' tacit knowledge, their skills, and seeds terms in order to converge to a common knowledge representation. Considering the scientific research management as a use case, we followed the proposed methodology to formalize our scientific research knowledge in the context of network and communication research field. Based on the generated ontology, we have developed a semantic web platform that allows collaboratively annotating experts' knowledge in a computer interpretable format that can be shared and reused by human and machines. Our evaluation is based on end users' quality of experience and feedbacks.
\end{abstract}

Keywords. Knowledge Sharing, Collaboration, Semantic Web, Ontology Development, Knowledge Management

\section{Introduction}

Research collaboration plays an important role in evaluating the research team outcomes [1, 2]. Successful collaborations take place if research team members and/or external partners can smoothly communicate based on a sharable knowledge that reflects the team skills [3]. Currently, scientific research team knowledge is embedded in unstructured documents including published articles, internal team reports, figures, tables and videos representing demonstrations. Thus,

${ }^{1}$ Corresponding author: Emna MEZGHANI, Email: emna.mezghani@laas.fr Telephone: +33561336921, Address: LAAS-CNRS, 7 avenue du Colonel Roche, 31400 Toulouse-France 
information is everywhere, heterogeneous and poorly organized which hamper knowledge exchange and sharing, and make information seeking is time-consuming.

Advances in Knowledge Management (KM) based on Information Technology (IT) provide important means to increase productivity and achieve the team effectiveness [4] since it provides methods and tools that capture, understand, share, and facilitate knowledge access and reuse by team members to create value [5-7]. However, IT support cannot cover more than $10-30 \%$ of KM [8]. More importantly, domain experts are the main knowledge source. Face-to-face interactions such as interviews, brainstorming, meetings, etc. are the keys for elucidating, capturing and sharing experts' tacit knowledge [9]. It is worth mentioning that the success of KM, especially which relies on the knowledge of experts, strongly depends on the acceptance of people involved in this process. At this end, human collaboration and participation is required to underpin an efficient knowledge transfer and sharing. Nevertheless, some cultural and social factors may hinder the progress of this process, especially if members may feel and think that knowledge sharing depletes the time and the efforts that can be invested in other activities more beneficial for themselves [10]. Moreover, each member has his/her own personality traits and characteristics, which makes managing a group of person complex, in particular if conflicts appear.

Consequently, to handle human barriers that may hamper extracting and sharing the experts' tacit knowledge, we propose a generic two-step methodology that describes processes and actors involved in the conceptualization and knowledge creation based on a multi-level approach. The objective of proposing a multi-level approach is to identify as a first stage a generic domain level that can be reused by any community/group of users interested to this domain, then extending it to support the user-requirements. We applied the proposed methodology to formalize and classify the scientific research activities within our SARA research team ${ }^{2}$.

The rest of the paper is organized as follow. Section 2 draws our proposed generic two-step methodology for experts' tacit knowledge conceptualization. Section 3 provides the results of applying the proposed methodology to formalize the tacit knowledge of our team experts. Section 4 presents our preliminary evaluation of the efficiency of the proposed methodology. Section 5 details existing researches dealing with collaborative scientific knowledge management and describes existing methodologies for building ontologies. Finally, section 6 summaries our contribution and identifies our future research works.

\section{A Generic Two-step Methodology for building Ontology}

Our methodology follows a top-down approach [11] which is mainly composed on two steps as shown in Figure 1: the "Knowledge Organization" that corresponds to conceptualizing and representing the knowledge in an appropriate format; and the "Knowledge Acquisition and Reuse" that allows the users collaboratively producing and consuming the knowledge. The "Knowledge Organization" represents the fundamental step. First, it identifies a core ontological model named Core Reference Ontology (CRO) describing the generic concepts and relations according to the formalized requirements. Then, it specializes these concepts to reflect the

\footnotetext{
${ }^{2}$ Services and Architectures for Advanced Networks- SARA: https://www.laas.fr/public/fr/sara
} 
domain experts' skills and knowledge based on their collaboration, and generates the Domain Specific Ontology (DSO). The main contribution of this methodology is to avoid conflicts and human barriers related to the personality characteristics when communicating with experts.

Based on an incremental approach, requirements are captured and refined with the collaboration of the leader expert. Thus, the key concepts of CRO, inference and inconsistency rules are identified $(1,2)$. After preparing the list of concepts and relations, the knowledge engineer identifies existing upper ontologies that can be reused and easily integrated with the $C R O$ (3). To personalize the $C R O$ in a specific domain, the domain experts are involved to enrich the $C R O$ with their own vision and tacit knowledge.

It is truism that each person has his/her specific character and manners to communicate and express his/her knowledge. Studies in psychology have shown that knowledge sharing behavior among individuals is influenced by personality traits [12]. According to Pervin [13], personality refers "to an individual's unique and stable pattern of thinking, feeling, acting and reacting to his or her social environment". These patterns, which explain why some individuals are motivated to share knowledge while others are not, can be classified according to the big five taxonomy [14]: extraversion that refers to energetic people who prioritize harmony in their social relationships; agreeableness that concerns friendly and cooperative people; conscientiousness that refers to reliable and responsible people; openness to experience that encompasses imaginative and flexible people who are interested in learning new experiences; and neuroticism that concerns people who may rapidly experience instable emotions such as nervosity and anxiety.

Accordingly, to avoid barriers related to social and psychological dimensions, our methodology adopts an incremental approach in which the identification of the knowledge structure is decomposed into sub-steps. First, experts are split into groups (4). Each group includes experts working on the same research area. Then, for each group, individual meetings with each expert are organized to discuss with him/her, to formalize his/her vision and to acquire as much as possible knowledge about his/her work $(5,6)$. By this way, problems such as being influenced by colleagues high in extraversion, and avoiding conflicts with colleagues high in neuroticism are covered. Furthermore, according to the expert character, different methods and questions are adopted to extract his/her knowledge. For example, the knowledge engineer reviews some relevant publications of the expert and extracts a set of keywords that help animating the discussion in order to deal with problems pertaining to people less in agreeableness or less in extraversion. At this stage, the $C R O$ is used when discussing with experts to converge to a unified model. By repeating the processes $(5,6)$, different models are generated. 


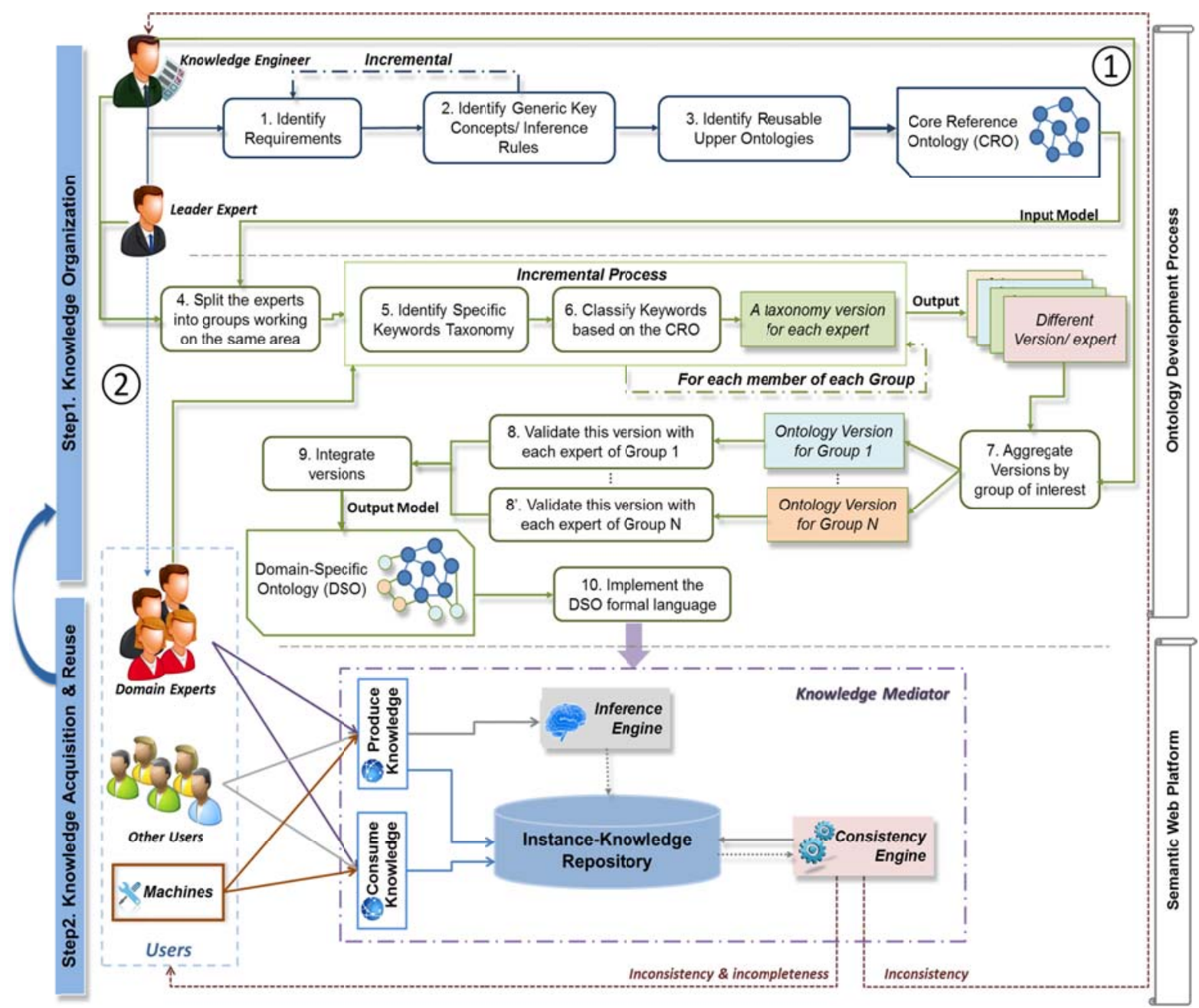

Figure 1. The generic two-step methodology for formalizing experts' tacit knowledge

Afterward, for each group, the different versions are aggregated (7) to provide a unified ontology schema which is approved and validated by the experts of this group (8, 8'). The final versions of all the groups are integrated in order to generate the Domain Specific Ontology (DSO) that reflects the team directions and interests, and formalizes its experts' visions (9).

During the design process, we used UML diagrams to model and visualize the ontology classes and relationships since it is accepted and understood by experts. The resulted ontology described in UML will be translated (10) into a formal ontology language such as OWL, RDF and RDFS to drive the knowledge acquisition process and enable reasoning for smart knowledge management. Our platform includes inference rules that automatically create new knowledge, and a Consistency Engine that detects knowledge inconsistency and incompleteness (e.g. noncompliance of cardinalities) based on domain rules. In particular, our platform relies on semantic web technology and provides a user friendly interface that enables a collaborative authoring and annotation among the team members. It is a kind of virtual knowledge mediator that interconnects team knowledge in a way that can be smoothly reused.

\section{Use Case Application: Scientific Research Management}


We followed our proposed methodology to formalize the scientific research domain. As a first stage, we identified the Scientific Research Activities Core Ontology (SRACO) that describes the generic concepts related to the scientific research management independent from the team interests. At a second stage, we specialized the SRACO to formalize the SARA research team skills and expertise in the context Network and Communication domain. The result of applying this methodology is the Network and Communication Research Domain Ontology (NCRDO) depicted in Figure 2. During the conceptualization phase, we have used the Astah Community tool $^{3}$ to represent the ontology classes and relationships as UML class diagram.

First, by collaborating with the research team leader, we identified the main administrative and high level scientific concepts such as the "Scientific Contribution", "Approach", "Method", "Technique", etc. Then, we identified possible existing upper ontologies that can be reused such as FOAF $^{4}$ ontology that describes the organization structure (e.g. foaf:Person and foaf:Organization), and the Event Ontology ${ }^{5}$ that describe the event:Event class. Based on an incremental approach, we aggregated the different identified concepts to generate SRACO. The completed version of the SRACO implemented in OWL can be downloaded from [15]. The next step is to personalize this ontology to capture the team experts' tacit knowledge and formalize their skills. Herein, our methodology intervenes to solve social issues that may appear with a group of persons. We split the experts into two groups: the "Network performance" and the "Distributed services and applications". Then, we individually met each expert and applied an incremental process to identify their seeds terms. Different visions and interpretations have been discussed and reformulated in order to converge to the specialized ontology (NCRDO) that details the scientific contributions criteria of the SARA team. Fortunately, our experts are with high conscientiousness and openness expressed through their reliability and their ability to help us aligning their knowledge with their colleague's experience in order to generate a unified ontology.

Figure 2 presents an excerpt of the main classes, relationships and cardinalities of the NCRDO. The specialized part is represented as sub-classes of the SRACO classes, which are represented in red color in Figure 2, except the "Research Directions" and the "Applicative Domain" are instances. For clarity reasons, we present only the "Applicative Domain" with the $<<$ instance $>>$ stereotype. A detailed OWL version of the NCRDO representing the SARA team interests can be downloaded from [16].

It is noteworthy that our proposed methodology is being used also in the medical domain to formalize the experts' medical knowledge and encode the disease management strategies, based on the collaboration of medical experts, in order to adapt the patient treatment plan [17].

\footnotetext{
${ }^{3}$ http://astah.net/editions/community (last visit March 17, 2015)

${ }^{4}$ FOAF: http://xmlns.com/foaf/spec/ (last visit March 19, 2015)

${ }^{5}$ Event ontology: http://motools. sourceforge.net/event/event.html\#term_classes (last visit March 19, 2015)
} 


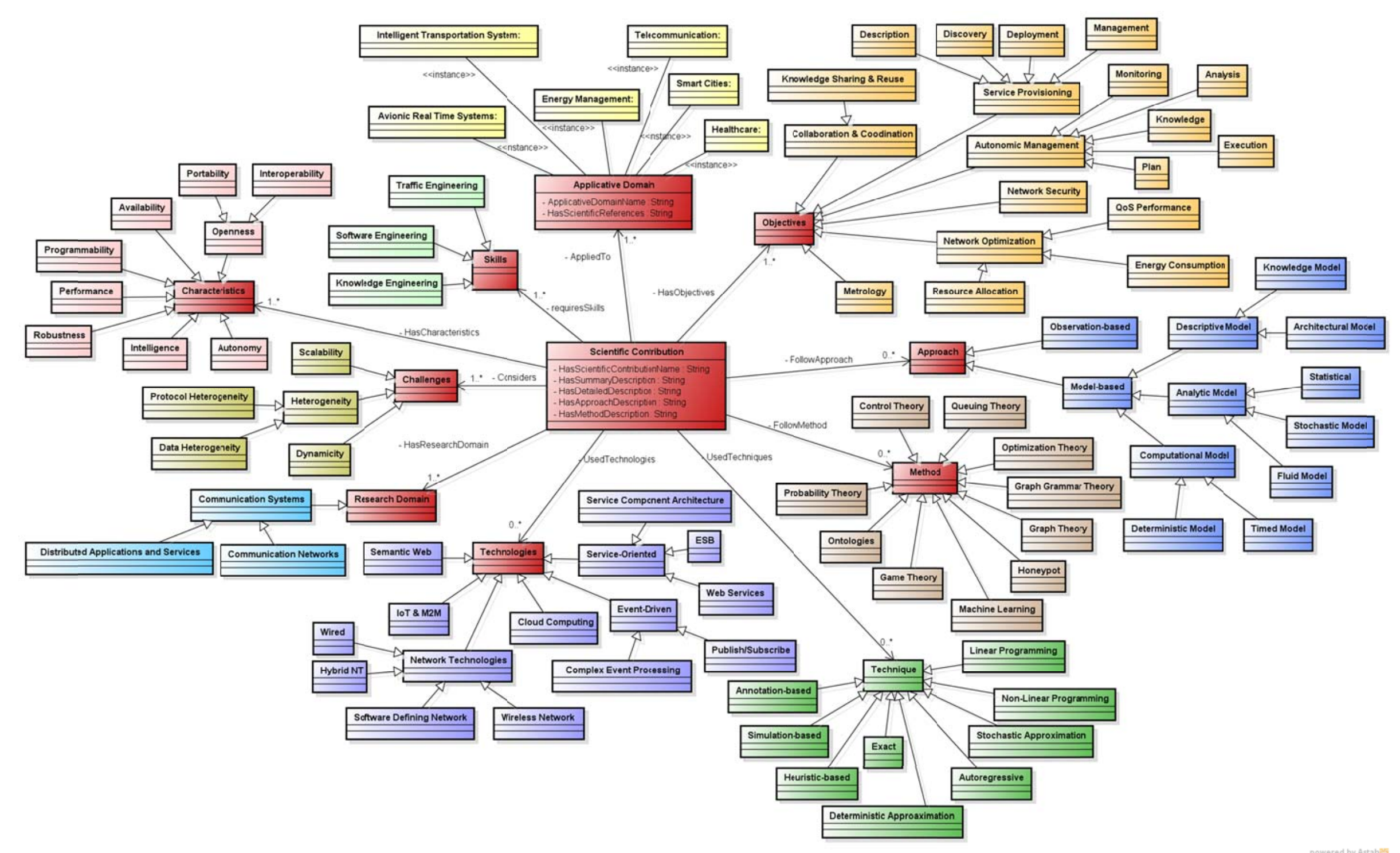

Figure 2. An excerpt of the main classes and relations of the Network and Communication Research Domain Ontology (NCRDO) 


\section{Evaluation}

To evaluate the efficiency of the proposed methodology, we developed a proof of concept implementing the Network and Communication Research Domain Ontology (NCRDO). We are interested in providing a scalable platform able to support the development of the personalized ontology and the collaborative knowledge annotation to provide the right seeds terms. Consequently, we selected Semantic MediaWiki (SMW) [18] as a semantic web technology since it unleashes the power of wikis for collaborative knowledge management, and ontologies for providing a common understanding of the domain. Moreover, SMW belongs to the "wiki for semantics" category of semantic wiki engines that support collaborative ontology development [19]. It offers mechanisms that map annotations embedded in wiki pages into a formal description using the OWL DL ontology language [20]. Thus, knowledge is collaboratively populated and can be seamlessly used by human and machines.

As a fist-line evaluation, we proposed three criteria that reflect the users' Quality of Experience $(\mathrm{QoE})$ presented in Figure 3. The first criterion is the completeness of the Domain Specific Ontology, which refers in this paper to the NCRDO. It allows evaluating the efficiency of our proposed methodology to extract as much as possible knowledge from the experts. Thus, the other research team members (PhD, PostDoc, Visitor, Trainee, etc.) can easily annotate their scientific contributions. The second criterion is more related to the openness of the platform to support the evolution of the NCRDO from both the meta-level and instance-level (e.g. adding new seeds terms with the respect of the SRACO schema). Finally, the third criterion refers to evaluating the human-machine interaction when (1) acquiring the knowledge and, (2) automatically generating new knowledge based on the semantic search mechanisms. As advocated in Figure 3, the evaluation provides a good sight about the proposed methodology in providing an ontology covering the main keywords of the team interests and directions.

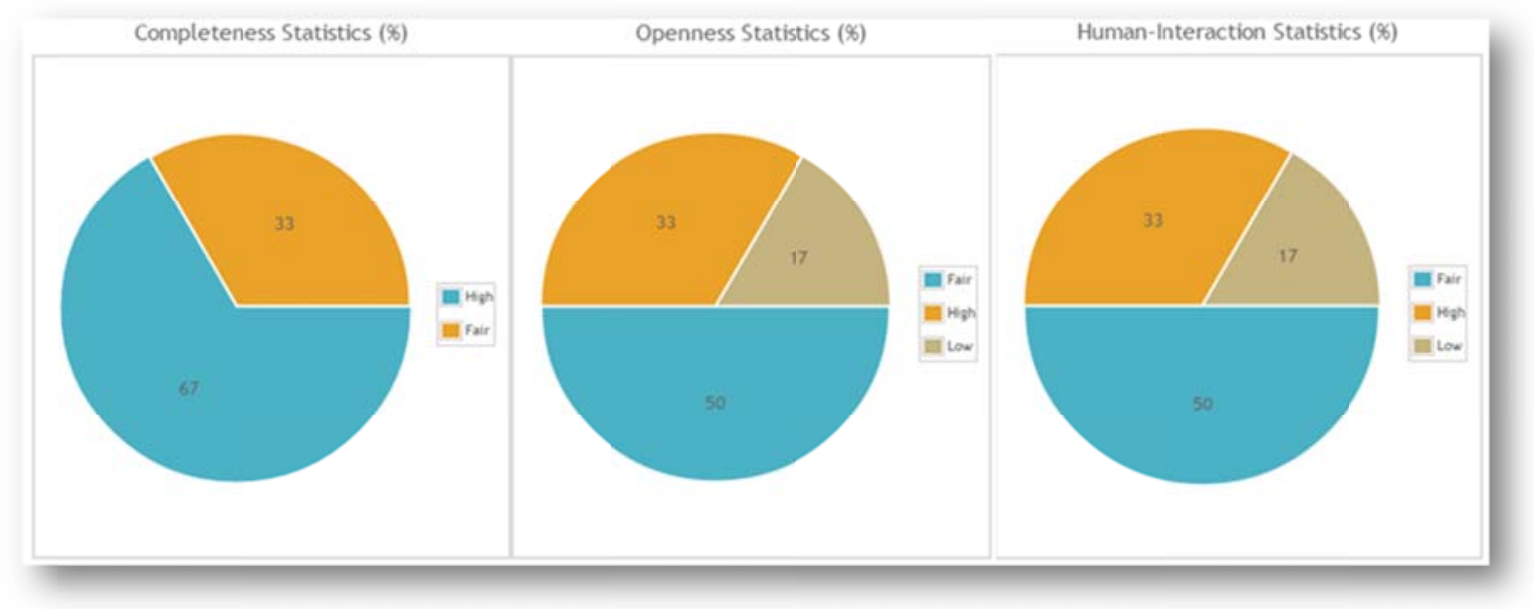

Figure 3. The Evaluation Results based on end users' feedbacks 


\section{Related Work}

Ontology has been widely used in different domains to ensure collaboration and provide common structure to knowledge for an easy share and reuse. Pirró et al.[21] described a framework implementing distributed ontology-based knowledge management systems within organization. The authors proposed a multi-level approach that identifies an upper ontology and a set of Core Ontologies Knowledge Entities describing the basic knowledge background of an organization, then deepens it to a particular domain in which an individual knowledge worker is interested. In the context of e-learning, Shih et al. [22] proposed an ontology-based approach to organize and retrieve learning content in geographically dispersed repositories. The main objective is to share and reuse data grids to solve the learning content management problem and provide information retrieval technique that enhances the resource discovery mechanism of data grids based on ontology. Similar to the work of $[21,22]$, we proposed a multi-level approach describing the knowledge to guarantee its modularity and reuse. However, none of [21, 22] identified the methodology used to develop the ontology. Our work proposed a generic methodology dedicated to tacit knowledge conceptualization and formalization using semantic web. Another interesting work is the work of Li et al. [23] where the authors proposed an ontology-centric architecture, which focuses on project characterization, then, specialized it to the phenomic domain where new classes were added to generate specific domain ontology. However in [23], the authors did not formalize the experts' skills which are considered in our work.

None of the discussed works $[21,22,23]$ clearly described how the knowledge is formalized and what methodology has been used to develop the ontology. In literature, many methodologies have been proposed for building ontology [24]. The most mature methodology is the METHONTOLOGY which was developed taking the IEEE 1074-1995 standard for software development process as a starting point [25]. It covers the ontology development process, the ontology life cycle and the techniques to achieve each activity [26] from the specification to the maintenance of the implemented ontology. Contrary to our proposed methodology, METHONTOLOGY does not provide information about how experts can be involved in this process to formalize their tacit knowledge. On-To-Knowledge [8] introduced the balance between human problem solving and the automated IT solutions. It starts with a feasibility study where problems and people that can be involved are identified. Then, the ontology requirements are specified, a semi-formal ontology description is elaborated, and finally the applicative ontology is produced and evaluated. Similar to On-To-Knowledge, our methodology points out the importance of addressing human issues in the ontology development process. However, our work details the workflow to manage these issues and interactions with experts.

Both METHONTOLOGY and On-To-Knowledge do not consider collaborative construction of ontologies [27] which is mandatory to formalize the experts' tacit knowledge. In this context, Kotis and Vouros [28] proposed a Human-Centered Ontology Engineering Methodology (HCOME). Compared to existing methodologies [24], HCOME contributes to involving the knowledge worker who focuses on developing and managing his/her ontology in his/her personal space. These ontologies are shared with other workers for review and evaluation. Thus, this methodology requires skills in ontology modeling. Similar to HCOME, our methodology is based on experts collaboration, but relies on face-to-face exchange, which remains more effective and exhibit higher performance results [29], when identifying the meta-knowledge reflecting experts' vision. $\mathrm{NeOn}[30]$ is a scenario-based methodology that adopts a different vision from existing methodologies. It does not define workflow for the ontology development, but identifies nine 
scenarios for collaboratively building ontologies and ontology networks, reusing and reengineering knowledge resources (ontological and non-ontological). Our methodology integrates some scenarios provided by $\mathrm{NeOn}$ such as reusing existing ontological knowledge sources; but it deepens the workflow for building ontologies and copes with social challenges that can loom up the collaborative ontology construction such as conflicts and psychological barriers.

\section{Conclusion}

In this paper, we proposed a generic two-step methodology dedicated to collaboratively conceptualizing and formalizing the experts' tacit knowledge within a team. This methodology takes into consideration the human barriers and adopts a multi-level approach to generate the core ontology describing generic concepts, and the domain specific ontology portraying the experts' skills. We presented the result of applying this methodology for managing the scientific research activities and we developed a semantic web platform to manage our team experts' tacit knowledge. The proposed methodology has been evaluated based on users' feedbacks and their quality of experience.

Currently, we are mainly working on automating the knowledge acquisition process by conceiving an intelligent information retrieval service that will contribute on the ontologyinstance evolution. Our future work will focus on the knowledge maintenance and reasoning.

\section{Acknowledgement}

We would like to thank all the SARA research team members, especially tenures for their collaboration to build the Network and Communication Research Domain Ontology and identify their interests.

\section{References}

[1] J.S. Katz, B.R. Martin, What is research collaboration?, Research policy, 26 (1997) 1-18.

[2] L. Tsui, S.A. Chapman, L. Schnirer, S. Stewart, A Handbook on Knowledge Sharing: Strategies and Recommendations for Researchers, Policy Makers and Service Providers, Community-University Partnership for the Study of Children, Youth, and Families Edmonton, 2006.

[3] C. Hussin, A. Razak, M. Hussain Alsayed, H. Mohamed Dahlan, Knowledge sharing in collaborative research activities, Journal of Information Systems Research and Innovation, 2 (2012) 1-8.

[4] R. Studer, V.R. Benjamins, D. Fensel, Knowledge engineering: principles and methods, Data \& knowledge engineering, 25 (1998) 161-197.

[5] M. Earl, Knowledge management strategies: Toward a taxonomy, Journal of management information systems, 18 (2001) 215-233.

[6] S.R. Ghani, Knowledge management: tools and techniques, DESIDOC Journal of Library \& Information Technology, 29 (2009) 33-38.

[7] R. Dieng, O. Corby, A. Giboin, M. Ribiere, Methods and tools for corporate knowledge management, International journal of human-computer studies, 51 (1999) 567-598.

[8] Y. Sure, S. Staab, R. Studer, On-to-knowledge methodology (OTKM), in: Handbook on ontologies, Springer, 2004, pp. 117-132.

[9] M. Alavi, D.E. Leidner, Review: Knowledge management and knowledge management systems: Conceptual foundations and research issues, MIS quarterly, (2001) 107-136.

[10] S. Wang, R.A. Noe, Knowledge sharing: A review and directions for future research, Human Resource Management Review, 20 (2010) 115-131.

[11] C. Roussey, F. Pinet, M. Kang, O. Corcho, An Introduction to Ontologies and Ontology Engineering, in: Ontologies in Urban Development Projects, Springer London, 2011, pp. 9-38.

[12] K. Yaakub, R. Shaari, S.A. Panatik, A. Rahman, Towards an understanding of the effect of core self-evaluations and knowledge sharing behaviour, International Journal of Applied Psychology, 3 (2013) 13-18.

[13] L.A. Pervin, Personality: Theory, assessment, and research, (1970). 
[14] A.T. Amayah, Knowledge Sharing, Personality Traits and Diversity: A Literature Review, in: Proceedings from The Midwest Research-to Practice Conference in Adult, Continuing, and Community Education. St. Louis, MO: USA, 2011.

[15] SRACO Ontology, Available at: http://homepages.laas.fr/emezghan/SRACO.owl .

[16] NCRDO Ontology, Available at: http://homepages.laas.fr/emezghan/NCRDO.owl.

[17] E. Mezghani, M. Da Silveira, C. Pruski, E. Exposito, K. Drira, A perspective of adaptation in healthcare, Studies in health technology and informatics, 205 (2014) 206-210.

[18] S. Schaffert, F. Bry, J. Baumeister, M. Kiesel, Semantic wikis, software, IEEE, 25 (2008) 8-11.

[19] F. Bry, S. Schaffert, D. Vrandečić, K. Weiand, Semantic wikis: Approaches, applications, and perspectives, Springer, 2012.

[20] M. Krötzsch, D. Vrandečić, M. Völkel, Semantic mediawiki, in: The Semantic Web-ISWC 2006, Springer, 2006, pp. 935-942.

[21] G. Pirró, C. Mastroianni, D. Talia, A framework for distributed knowledge management: Design and implementation, Future Generation Computer Systems, 26 (2010) 38-49.

[22] W.-C. Shih, C.-T. Yang, S.-S. Tseng, Ontology-based content organization and retrieval for SCORM-compliant teaching materials in data grids, Future Generation Computer Systems, 25 (2009) 687-694.

[23] Y.-F. Li, G. Kennedy, F. Ngoran, P. Wu, J. Hunter, An ontology-centric architecture for extensible scientific data management systems, Future Generation Computer Systems, 29 (2013) 641-653.

[24] Y. Sure, S. Staab, R. Studer, Ontology engineering methodology, in: Handbook on ontologies, Springer, 2009, pp. $135-152$.

[25] M. Fernández-López, Overview of methodologies for building ontologies, in: IJCAI99 Workshop on Ontologies and Problem-Solving Methods: Lessons Learned and Future Trends, Stockholm, 1999.

[26] M. Fernández-López, A. Gómez-Pérez, N. Juristo, METHONTOLOGY: From Ontological Art Towards Ontological Engineering, AAAI Symposium on Ontological Engineering, Stanford, 1997.

[27] O. Corcho, M. Fernández-López, A. Gómez-Pérez, Methodologies, tools and languages for building ontologies. Where is their meeting point?, Data \& knowledge engineering, 46 (2003) 41-64.

[28] K. Kotis, G.A. Vouros, Human-centered ontology engineering: The HCOME methodology, Knowledge and Information systems, 10 (2006) 109-131.

[29] M.E. Warkentin, L. Sayeed, R. Hightower, Virtual Teams versus Face-to-Face Teams: An Exploratory Study of a Web-based Conference System*, Decision Sciences, 28 (1997) 975-996.

[30] M.C. Suarez-Figueroa, A. Gomez-Perez, M. Fernandez-Lopez, The NeOn methodology for ontology engineering, in: Ontology engineering in a networked world, Springer, 2012, pp. 9-34. 